\title{
An Analogue Model Study of Ocean-Wave Induced Magnetic Field Variations Near a Coastline
}

\author{
T. Miles and H. W. Dosso \\ Department of Physics, University of Victoria, \\ Victoria, B. C., Canada \\ (Received October 7, 1977; Revised January 12, 1978)
}

\begin{abstract}
A laboratory analogue model, described previously by Miles et al. (1977), was used to study the effect of a sloping ocean floor with a shelf, and a step with a shelf, on the magnetic variations induced by ocean waves moving in a static magnetic field. The model measurements indicate that for a shallow ocean, the amplitudes of the magnetic variations are attenuated as the wave travels over the wedge, reach a minimum at the shelf edge, then again increase over the shelf. For the case of the step with a shelf maximum attenuation occurs at the shelf edge followed by an enhancement over the shelf. For both cases, the depth of the fluid over the shelf is the important factor in determining the behaviour of the induced field.
\end{abstract}

\section{Introduction}

The induction of magnetic fields by ocean waves in an infinite depth ocean or in a uniform finite depth ocean has been studied theoretically by many authors, including Young et al. (1920), Crews and Futterman (1962), Weaver (1965), Groskaya et al. (1972), LARSEN (1973), Preisendorfer et al. (1974), Klein et al. (1975), and Podney (1975). Induction problems involving an ocean with a sloping or an irregular bottom, or an ocean with a coastline, have not as yet been solved analytically nor treated by numerical techniques. One method of studying these problems involves the aid of a suitably scaled laboratory analogue model. The present work deals with a laboratory analogue model study of fields induced by waves over a shelving sea floor and shelf, and over a vertical step and shelf.

\section{Model Measurements and Discussion of Results}

The analogue model scaling conditions used in the present work were developed and described earlier (MILES et al., 1977) and will not be treated here. In the same work, the model construction, the experimental techniques, as well as the validity of the model results for a finite depth model ocean, were discussed in detail.

In the present work, the scaling factors for conductivity, linear dimension, and frequency are respectively $\sigma^{\prime} / \sigma=4.33 \times 10^{-6}, d^{\prime} / d=3.77 \times 10^{3}, f^{\prime} / f=1.60 \times 10^{-2}$, where primed quantities refer to geophysical parameters and unprimed to model parameters. Using these scaling factors, $1 \mathrm{~cm}$ in the model corresponds to $38 \mathrm{~m}$ in the ocean, a wave- 
length of $9.5 \mathrm{~cm}$ in the mercury simulates a wavelength of $358 \mathrm{~m}$ in the ocean, and a frequency of $4 \mathrm{~Hz}$ in the model simulates a frequency of $0.064 \mathrm{~Hz}$ in the ocean. For the wavelength of $\lambda=9.5 \mathrm{~cm}$, the mercury depth of $2.8 \mathrm{~cm}$ was less than $1 / 3 \lambda$. The steady primary field used in the model was 57 gauss $\left(5.7 \times 10^{-3} T\right)$. Waves in the mercury were generated by dropping a small horizontal lucite rod into the mercury from a fixed height of $0.5 \mathrm{~cm}$. This wave generator was situated $5 \mathrm{~cm}$ from one end of the mercury tank. The magnetometer probe, used to measure the induced field, was positioned so as to be sensitive to the horizontal induced field, as measured in a direction normal to the wave front in the mercury. The probe, mounted on a rail, could be positioned very accurately. The height of the probe above the mercury was $0.6 \mathrm{~cm}$. This corresponded to a height of 23 meters above the surface of the ocean.

Measurements of the induced field were carried out point by point for traverses over

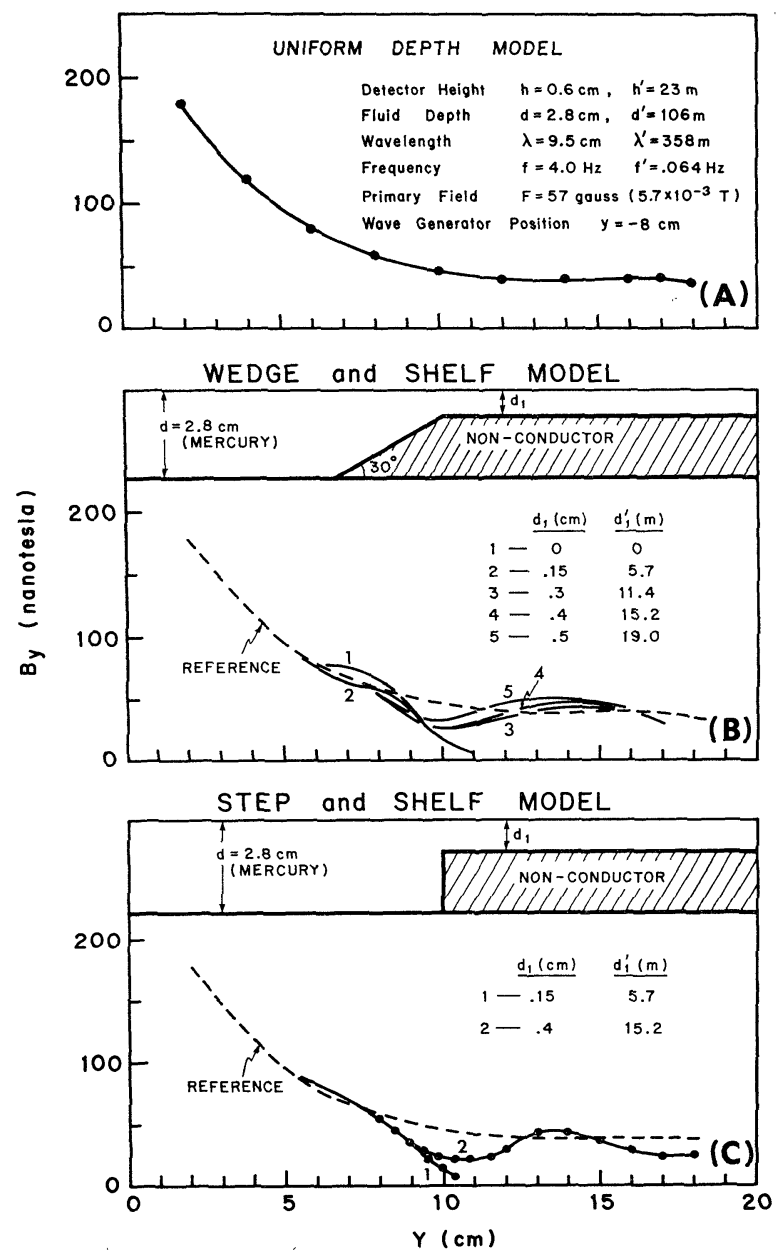

Fig. 1. Model magnetic field variations induced by fluid surface waves moving in a static magnetic field. 
the model ocean for the cases of a uniform depth ocean, a sloping sea floor and shelf, and for a vertical step and shelf in the sea floor. The induced fields for these cases are shown in Fig. 1.

The induced field for a traverse over the uniform depth ocean is shown in Fig. 1(a). With the wave source located at $y=-8 \mathrm{~cm}$, it is seen that the induced field initially falls off rapidly with distance from the source then becomes essentially uniform in the neighbourhood of $y=10 \mathrm{~cm}$. The curve of Fig. 1(a) is used as a reference (dashed line) in Figs. 1(b) and (c).

To simulate a sloping ocean floor and a shelf near a coastline, a non-conducting (lucite) $30^{\circ}$ wedge was positioned in the mercury as shown at the top of Fig. 1(b). With the uniform depth region held at a constant depth $d=2.8 \mathrm{~cm}$, measurements were made for various wedges with shelf depths $d_{1}$. The curves in this diagram show the magnetic field amplitudes for five different wedges and the curves labelled 1-5 correspond to shelf depths $d_{1}=0,5.7,11.4,15.2,19.0$ meters. It is to be noted for curve 1 that the amplitude of the induced field builds up over the ramp, becoming a maximum approximately half way up and then falls off sharply as the ocean depth decreases further. The enhancement begins just beyond the point towards the conductor where the wedge meets the ocean bottom. Somewhat similar behaviour, but without enhancement, is observed for the very shallow layer for curve 2 , with the field falling off sharply again. For each of the other 3 curves corresponding to shelf depths of 11.4, 15.2, 19.0 meters, essentially no enhancement occurs over the ramp, but there is an attenuation with a field minimum directly over the edge of the shelf. The field then builds up over the shelf, attaining a value slightly greater than the reference field. The minimum value over the shelf edge decreases as the ocean depth over the shelf increases. As the depth $d_{1}$ is increased further, the fields should approach the reference values. The attenuation at the shelf edge, and the build up of the field beyond the shelf edge can be explained in part as due to the modification of the waves as they pass over the slope from the uniform depth ocean to the shallow ocean, and in part in terms of the changing induced current distribution with changing ocean depth. The increasing wave amplitude for a range of decreasing fluid depths would tend to enhance the field. Further, the interaction with the obstacles (wedge and step) may lead to interference patterns in the wave motion over the shelf, resulting in further induced field anomalies.

In Fig. 1(c) we see results for the case of a step in the ocean floor followed by a shelf for two depths $d_{1}=0.15 \mathrm{~cm}$ and $d_{1}=0.4 \mathrm{~cm}$. For this model, a very small enhancement occurs as the wave approaches the vertical interface, the field falls off rapidly as the interface is approached for both curves. For the $0.4 \mathrm{~cm}$ depth, the broad attenuation has a minimum approximately over the interface, the minimum value being essentially the same as we saw for the case of the wedge for corresponding depth $d_{1}=0.4 \mathrm{~cm}$. The amplitude then increases to a maximum at approximately $3^{1} / 2 \mathrm{~cm}$ beyond the interface corresponding to approximately $1 / 3 \lambda$ beyond the interface, and then falls off to values below the reference value. Again, it is expected that as $d_{1}$ is increased further the fields should approach the reference curve values.

Measurements for further depths $d_{1}$ for the wedge and step cases will be carried out and presented at a later date. Other cases of a non-uniform sea floor, including sea 
mounts and dykes, too can readily be studied. In conclusion, the present results indicate that the wave motion and the induction mechanism is far from simple for the case of the non-uniform ocean floor, and that further studies are warranted.

\section{REFERENCES}

CREws, A. and J. Futterman, Geomagnetic micropulsations due to the motion of ocean waves, $J$. Geophys. Res., 67, 299-306, 1962.

Groskaya, Ye. M., R. G. Skrynnikov, and G. V. Sokolov, Magnetic field variations induced by the motion of sea waves in shallow water, Geomagn. Aero., 12, 131-134, 1972.

KLein, M., P. Louvet, and P. Morat, Measurement of electromagnetic effects generated by swell, Phys. Earth Planet. Inter., 10, 49-54, 1975.

LARSEN, J. C., An introduction to electromagnetic induction in the ocean, Phys. Earth Planet. Inter., 7, 389-398, 1973.

Miles, T., H. W. Dosso, and T. P. NG, An analogue model for studying magnetic variations induced by ocean waves, Phys. Earth Planet. Inter., 14, 137-142, 1977.

PoDney, W., Electromagnetic fields generated by ocean waves, J. Geophys. Res., 80, 2977-2990, 1975.

Preisendorfer, R. W., J. C. Larsen, and M. A. Sklarz, Electromagnetic fields induced by planeparallel internal and surface ocean waves, Report No. HIG-74-8, Hawaii Institute of Geophysics, University of Hawaii, 1974.

WeAVER, J. T., Magnetic variations associated with ocean waves and swell, J. Geophys. Res., 70, 1921$1929,1965$.

Young, F. B., H. Gerrard, and W. Jerons, On electric distrurbances due to tides and waves, Phil. Mag., 40, 149-159, 1920. 